\title{
Inclusión educativa, habilidades diferentes y asperger
}

Mg. Abel Cuzcano Zapata

Mg. César Ruiz Alva

\section{INTRODUCCIÓN}

Actualmente en el sistema escolar peruano no se cumplen de manera integral, las políticas que apoyan en forma efectiva a los estudiantes con necesidades especiales entre ellas a los alumnos con Trastornos generalizados del Desarrollo, como es el caso de los niños y jóvenes con Diagnóstico de Asperger.

La Ley de las Personas con Discapacidad, Ley № 29973, que rige a las instituciones públicas y privadas, indica que todas las instituciones educativas, en todos los niveles y modalidades, deben reservar al menos dos vacantes por aula para estudiantes con necesidades educativas especiales asociadas a discapacidad (leve o moderada) y el Director debe solicitar apoyo al SAANE(Servicio de apoyo y asesoramiento a las necesidades Educativas Especiales) a fin de garantizar la atención educativa pertinente, de estos estudiantes, sin embargo la realidad es que en los colegios en general no hay un programa de inclusión educativa, ni políticas institucionales de inclusión, ni adaptaciones curriculares adecuadas, el personal no tiene entre sus funciones, la atención a la diversidad.

Se suma a todo esto que el Ministerio de Educación, ha prohibido la evaluación psicopedagógica de ingreso de los niños que postulan a los colegios y muchos de ellos presentan problemas de aprendizaje u otras dificultades más serias, como los TDAH, Asperger, Trastornos de conducta, entre otros de las que ni los padres, ni el colegio son conscientes, lo que no les permite el diagnóstico pertinente, eficaz, temprano y preventivo.

Según la Ley General de discapacitados dice en su Artículo 1. "La presente Ley tiene la finalidad de establecer el marco legal para la promoción, protección y realización, en condiciones de igualdad, de los derechos de la persona con discapacidad, promoviendo su desarrollo e inclusión plena y efectiva en la vida política, económica, social, cultural y tecnológica" por lo que se espera que los colegios implementen Servicios de Educación Inclusiva para atender de manera óptima a alumnos 
con dificultades o discapacidades que responda a las necesidades planteadas y poder cumplir el objetivo que plantea el Ministerio de Educación en sus objetivos generales, que la educación peruana para el 2021 debe conseguir : "Oportunidades y resultados educativos de igual calidad para todos".

Es en la década del 90 que se desarrolló el Proyecto de Integración de Niños con Necesidades Especiales a la Escuela Regular, con el asesoramiento de la UNESCO, incorporándose por primera vez los estudiantes con discapacidad a los colegios regulares. En este contexto se formuló la nueva Ley General de Educación, Ley № 28044 promulgada en julio del 2003, en la que "la educación inclusiva emerge como respuesta ante las culturas y prácticas tradicionales asociadas a un modelo clínico, rehabilitador, basado en la patología, para promover una comunidad y una escuela que acoja a todos los estudiantes, sustentada en el planteamiento de atención a la diversidad en que las instancias del sector a nivel nacional, regional, local y las instituciones educativas pueden atender al conjunto de estudiantes con todas sus diferencias y en cualquier circunstancia. Esta legislación educativa aborda el derecho a la educación de los estudiantes con discapacidad bajo una concepción de educación inclusiva, transversal al sistema educativo, explicitando entre otros principios la calidad y la equidad, y cuyos marcos orientadores fueron planteados en los reglamentos de los diferentes niveles, modalidades y formas educativas, detallándose aspectos fundamentales para proponer una respuesta educativa pertinente". Pero falta mucho por hacer, por ejemplo que en los planes de estudio de las Universidades especialmente de carreras como Educación y Psicología se incluyan materias sobre las personas con Habilidades diferentes para que su atención sea sobre bases científicas.

Si bien en el país se vienen dando algunos avances, en relación a las políticas educativas hacia las personas con discapacidad, se necesita aún que se clarifique sus competencias y responsabilidades, muchas de ellas expresadas en los reglamentos de la Ley General de Educación, para su aplicación en el sistema educativo.

\section{LOS ALUMNOS ASPERGER Y LOS TRASTORNOS GENERALIZADOS DEL DESARROLLO (TGD)}

La primera persona en utilizar el término Síndrome de Asperger fue Lorna Wing, quien recogió los escritos y las investigaciones de Asperger en el artículo titulado "El Síndrome de Asperger: un relato clínico", publicado en 1981. Lorna Wing identificó tres grandes áreas afectadas, las cuales dieron lugar a la denominada Triada de Wing, siendo estas áreas: 1) competencia de relación social, 2) comunicación, 3) inflexibilidad mental y comportamental.

Los TGD hacen alusión a una perturbación grave y generalizada de varias áreas del desarrollo como los trastornos de la relación social, los trastorno de la comunicación (expresión y comprensión del lenguaje) y falta de flexibilidad mental con comportamientos, intereses y actividades estereotipadas. Los alumnos con Asperger caen dentro de los Trastorno del Espectro Autista (TEA), ya que recoge un cuadro clínico que no es uniforme, que oscila en un espectro de mayor a menor afectación; varía con el tiempo y se encuentra influido por otros factores como el funcionamiento intelectual, el nivel comunicativo, las alteraciones de conducta como los más relevantes. 
Gilberg (2010) señala que el síndrome de Asperger se aplica a niños, adolescentes y adultos que evidenciando una inteligencia normal (de preferencia $\mathrm{Cl}$ de 85 o más alto), y no habiendo tenido un retraso significativo en el desarrollo del lenguaje, presentan dificultades en la interacción y flexibilidad social, tienen intereses restringidos y predilección por las actividades rutinarias o repetitivas. Es la forma más leve de los 'trastornos del espectro autista' y está presente aproximadamente en una de cada 200 personas, varones en su gran mayoría; suele haber historia de SA en la familia, generalmente en la rama paterna.

La definición ha ido variando con el paso del tiempo, así pues en el Manual Diagnóstico y Estadístico de Trastornos Mentales DSM-IV y en la Clasificación Internacional de Enfermedades CIE el síndrome de Asperger, se incluye en la categoría de trastornos generalizados del desarrollo. Sin embargo en el reciente DSM-V pasa a considerarse como un trastorno del espectro autista. Esta patología afecta a niños, adolescentes y personas adultas y quienes lo padece poseen un coeficiente intelectual normal 0 incluso superior a la media sin embargo no son capaces de relacionarse adecuadamente

\section{PRINCIPALES TEORÍAS QUE EXPLICAN EL ASPERGER}

Cobo(2015) (citado por Cuzcano 2016) , señala que entre las principales teorías que explican el Asperger tenemos:

\section{LA TEORÍA DE LA MENTE}

Hace referencia a la capacidad que tenemos las personas de establecer una representación interna de nuestros estados mentales, y la de los demás, tales como son los deseos, creencias e intenciones. Esa representación mental e interna que formamos, la adquirimos de manera innata, de modo que somos capaces de interpretar ciertas situaciones de una manera automática en cambio las personas que se encuentren dentro de un TEA, tienen que aprender estos patrones sociales ya que por la simple observación son incapaces de adquirirlos. En este sentido esta teoría propuesta por Simon Baron-Cohen, Alan Leslie y Uta Frith, explica las dificultades que presentan las personas con Síndrome de Asperger al no ser capaces de comprender ciertas situaciones que no quedan explicitas en determinadas situaciones sociales tales como las siguientes: predecir ciertas conductas en los demás, interpretar el doble sentido en una conversación, así como a no comprender las bromas, chistes, la ironía, poseen una baja reciprocidad emocional y poca sensibilidad a las señales sociales.

Algunas de las limitaciones para comprender el mundo mental de los demás sería la siguiente: - Dificultad para predecir la conducta de los demás. - Problemas para darse cuenta de las intenciones de los demás. - Dificultad a la hora de explicar sus conductas. - Problemas en la comprensión de cómo sus conductas pueden afectar a los demás. • Incapacidad para darse cuenta del nivel de comprensión e interés de las personas que están escuchando cuando ellos están hablando, lo que a veces conlleva a que utilicen conversaciones tipo monólogo. • Dificultades en la anticipación. - Dificultad en narrar la memoria autobiográfica. - Dificultades p ara mentir y para comprender engaños. - Dificultad para comprender el uso de reglas en un acto comunicativo, no suelen respetar turnos, no mantienen un contacto ocular adecuado. 
Estos autores proponen que las dificultades que estas personas presentan en el aspecto social, la comunicación e imaginación, lo que se ha denominado como la triada de Wing, es debido a una dificultad o trastorno de la capacidad para "leer o entender la mente".

\section{TEORÍA DEL DÉFICIT EN FUNCIÓN EJECUTIVA}

Las funciones ejecutivas suelen definirse como un conjunto de operaciones cognitivas que son las responsables de activar una serie de estrategias de resolución de problemas con el propósito de alcanzar una meta. Estas conductas están mediadas por los lóbulos frontales, los cuales están encargados de actividades tales como atención, memoria de trabajo, planificación, organización, ejecución, finalización, evaluación tareas y actividades e inhibición de respuestas inadecuadas. Esta teoría intenta explicar una serie de limitaciones que se observan en el comportamiento diario de personas con síndrome de Asperger:

Déficit en organización: Presentan dificultades a la hora de secuenciar los pasos que les permiten solucionar un problema. - Déficit en planificación; Dificultades para hacer planes y luego seguir los planes que guían el comportamiento. • Déficit en Atención: Los problemas de atención son muy comunes en las personas con Síndrome de Asperger, ya que suelen mostrar una atención selectiva a los estímulos de interés. Además suelen presentar problemas en discriminar los estímulos relevantes de los irrelevantes. - Déficit de motivación en el aprendizaje: Existe una escasa motivación hacia el aprendizaje en las personas con Síndrome de Asperger cuando tienen que aprender aspectos que no tienen interés para ellos. - Inhibición de respuesta: Las personas con Síndrome de Asperger suelen tener resistencia a interrumpir una conducta en el momento adecuado. • Generalización: Los sujetos con Síndrome de Asperger suelen presentar dificultades para aprender estrategias de resolución de problemas, así como problemas de abstracción sobre los principios que regulan las soluciones a los mismos. Además cuando se encuentran en una situación nueva la ausencia de generalización suele provocar frustración y el abandono de la tarea que estén realizando. • Suelen utilizar un razonamiento de forma concreto en lugar de abstracto, utilizan un razonamiento literal a pesar de tener una inteligencia normal. Memoria de trabajo: Suelen tener problemas a la hora de recordar los pasos que siguen a la ejecución de una actividad. - Rigidez mental: Carencia de flexibilidad para adoptar diferentes perspectivas sobre la misma situación en diferentes momentos. Además suelen tener excesiva preocupación 23 por determinados temas y eso les restringe su capacidad para centrarse en otras actividades o temas de conversación. Tienden a monopolizar los temas de conversación según sus propios intereses. - Control conductual: Poseen un escaso control del rendimiento mientras realizan una actividad.

\section{TEORÍA DEL DEFICIT EN COHERENCIA CENTRAL}

Uta Frith y Francesca Happé han estudiado el procesamiento de la información y el perfil lingüístico de estos chicos y proponen que tanto el Autismo como el Síndrome de Asperger se caracterizan por una débil coherencia central. Con el término de coherencia central hacen referencia la tendencia humana de procesar la información de una manera global y contextual, de este modo cuando por ejemplo, leemos 24 una historia realizamos una pequeña abstracción, nos quedamos con los aspectos más significativos y generales; somos capaces de recordar información general y no 
recordamos tanto los detalles. Attwood dice que: "Una metáfora muy útil para entender en qué consiste la coherencia central débil es imaginarnos que enrollamos una hoja de papel en forma de tubo y con un ojo cerrado lo aplicamos contra el otro ojo abierto, como si fuera un telescopio, y miramos el mundo a través de él: se ven los detalles pero no se percibe el contexto". (Attwood, 2007:391).

Esta teoría propuesta por Uta Frith, señala que las personas que tienen un trastorno del espectro autista poseen un procesamiento centrado en los detalles y en las partes de un objeto o de una historia; se olvidan de la imagen o estructura global y no integran la información del contexto para buscar un sentido general y coherente. Como consecuencia de este tipo de procesamiento, la información que se adquiere posee un significado descontextualizado.

\section{DISFUNCIÓN DEL HEMISFERIO DERECHO}

En el Síndrome de Asperger encontramos una serie de rasgos centrales compatibles con una disfunción en el hemisferio derecho. El hemisferio cerebral izquierdo es el encargado del procesamiento y producción del lenguaje, así como de procesar mejor la información que proviene por vía auditiva. En cambio el hemisferio cerebral derecho se encarga del procesamiento de la información viso-espacial y de numerosos aspectos de la comunicación no verbal. Este hemisferio del cerebro es capaz de analizar la información de una forma holística y global, teniendo en cuenta el contexto, interpretando adecuadamente los gestos, el tono de voz y la expresión facial del interlocutor. Este hemisferio derecho procesa mejor la información táctil y visual. Disfunciones en el hemisferio derecho puede conllevar: - Dificultad para comprender la comunicación no verbal (entonación, volumen del habla, gestos y ademanes faciales y corporales). - Presencia de respuestas emocionales exageradas o poco coherentes con el contexto. - Dificultad para adaptarse a situaciones nuevas (rigidez). • Limitadas habilidades de organización viso-espacial. - Bajo rendimiento en tareas que requieran integración de la información viso-motora - Pocas habilidades de relación social. Desde esta perspectiva (V. Klin et. Al, 1995) se defiende que algunos de los síntomas principales del Síndrome de Asperger están relacionados con una disfunción de la parte derecha del cerebro. Esta hipótesis explicaría la superioridad de estos niños en CI Verbal frente al Cl Manipulativo, así como las dificultades que presentan muchas personas con Síndrome de Asperger.

Los diferentes enfoques deben estar orientados a ofrecer un modelo de atención educativa de atención a la diversidad, sobre la base de principios fundamentales de equidad, normalización, inclusión y el término de necesidades específicas de apoyo educativo que garantice el acceso y la permanencia en el sistema educativo del alumnado con necesidades específicas de apoyo educativo.(Alcantud 2003).

\section{SÍNTOMAS DEL SÍNDROME DE ASPERGER EN LOS NIÑOS}

Ruiz (2015) refiere que los niños con Síndrome de Asperger se caracterizan por tener áreas de especial interés peculiares e idiomáticas. Se interesan en áreas intelectuales específicas como las matemáticas, los temas científicos o la historia, la geografía, los mapas, la astronomía, los aviones 
o los trenes. Estos intereses pueden cambiar con el tiempo, pero otras veces persisten y en la edad adulta constituyen la base para una profesión. El diagnóstico preciso y seguro solo podrá realizarlo un especialista, así como su tratamiento. Sin embargo, existen algunas características que pueden observar los padres cuando sus hijos tienen entre 2 y 7 años de edad para identificar el Síndrome de Asperger.

Normalmente, un niño con Síndrome Asperger suele presentar algunas de estas características:

\section{Habilidades sociales y control emocional}

- No disfruta normalmente del contacto social. Se relaciona mejor con adultos que con los niños de su misma edad. No se interesa por los deportes.

- Tiene problemas al jugar con otros niños. No entiende las reglas implícitas del juego. Quiere imponer sus propias reglas, y ganar siempre. Tal vez por eso prefiera jugar sólo.

- Le cuesta salir de casa. No le gusta ir al colegio. Y presenta conflictos con sus compañeros.

- Le cuesta identificar sus sentimientos y de los demás. Presenta más rabietas de lo normal. Llora con facilidad, por todo.

- Tiene dificultades para entender las intenciones de los demás. Es ingenuo. No tiene malicia. Es sincero

\section{Habilidades de comunicación}

- No suele mirarte a los ojos cuando te habla. Se cree en todo aquello que se le dice, y no entiende las ironías. Se interesa poco por lo que dicen los demás. Le cuesta entender una conversación larga, y cambia de tema cuando está confuso.

- Habla mucho, en un tono alto y peculiar, y usa un lenguaje pedante, extremamente formal y con un extenso vocabulario. Inventa palabras o expresiones idiosincrásicas.

- En ocasiones parece estar ausente, absorto en sus pensamientos.

\section{Habilidades de comprensión}

- Siente dificultad de entender el contexto amplio de un problema. Le cuesta entender una pregunta compleja y tarda en responder.

- A menudo no comprende una crítica o un castigo. Así como no entiende el por qué debe portarse con distintas formas, según una situación social.

- Tiene una memoria excepcional para recordar datos y fechas.

- Tiene especial interés por las matemáticas y las ciencias en general.

- Aprende a leer solo a una edad temprana.

- Demuestra escasa imaginación y creatividad, por ejemplo, para jugar con muñecos.

- Tiene un sentido de humor peculiar.

\section{Intereses específicos}

- Cuando algún tema en particular le fascina, ocupa la mayor parte de su tiempo libre en pensar, hablar o escribir sobre el asunto, sin importarse con la opinión de los demás . 
- Repite compulsivamente ciertas acciones o pensamientos para sentirse seguro .

- Le gusta la rutina. No tolera los cambios imprevistos. Tiene rituales elaborados que deben ser cumplidos.

\section{Habilidades de movimiento}

- Posee una pobre coordinación motriz. Corre a un ritmo extraño, y no tiene destreza para atrapar una pelota.

- Le cuesta vestirse, abrocharse los botones o hacer un lazo con los cordones.

\section{Otras características}

- Miedo, angustia debido a sonidos como los de un aparato eléctrico

- Ligeros roces sobre la piel o la cabeza

- Tendencia a agitarse o mecerse cuando está excitado o angustiado

- Falta de sensibilidad a niveles bajos de dolor

- Tardanza en adquirir el habla, en pocos casos

- Muecas, espasmos o tics faciales inusuales

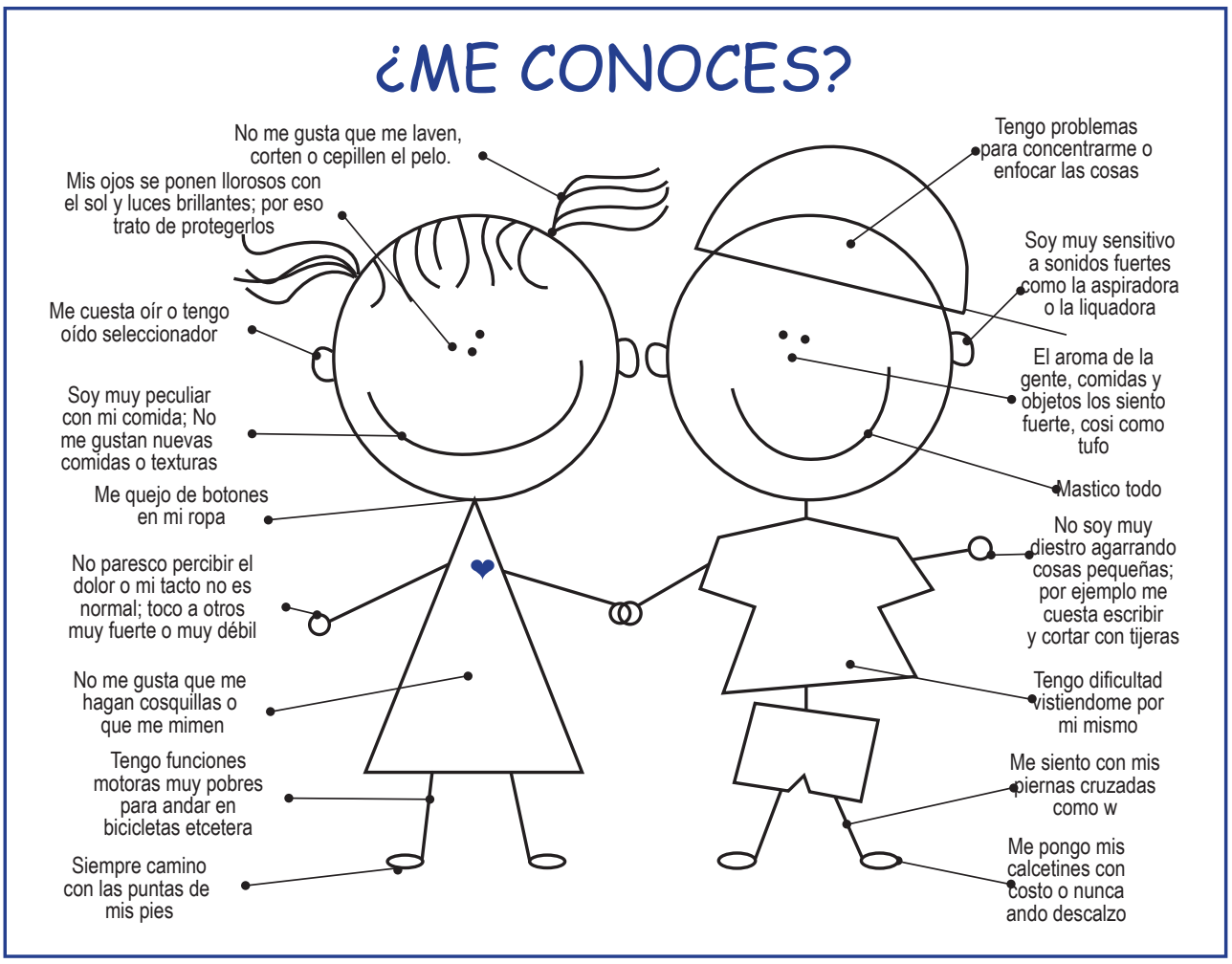




\section{ROL DEL MAESTRO}

En un sistema educativo inmerso en una sociedad democrática, la atención a la diversidad es un derecho irrenunciable. En la actualidad, el profesorado se encuentra con una heterogeneidad en el alumnado al que está obligado a atender. En la mayoría de las ocasiones, dicha diversidad puede ser atendida desde la acción tutorial inherente a la función docente; sin embargo, en otras ocasiones los profesionales de la educación deben de hacer uso de otro perfil profesional para poder dar la respuesta educativa adecuada a un alumno o alumna. La atención al alumnado con trastornos del desarrollo en el aula ordinaria, sin engañarnos, es un camino que estamos comenzando a recorrer no exento de obstáculos. Con la finalidad de orientar a los docentes que se enfrentan con el trabajo diario de enseñar y educar al alumnado que presenta Síndrome de Asperger se presentan las siguientes pautas generales de intervención. Sin olvidar que cada caso es único e irrepetible y que requiere la evaluación de los profesionales de la salud.

\section{Siguiendo a Thomas(2002) brindamos algunas consideraciones y estrategias útiles para la atención del Asperger desde el aula:}

1.- Entender a los alumnos: comprender su funcionamiento psicológico y su procesamiento neurológico, los desafíos que les plantea el aprendizaje, y transformar esta comprensión en estrategias eficaces para mejorar la enseñanza y la atención que se les brinda.

2.- Hablar menos cuando el alumno está estresado o disgustado.

3.- Avisar a los alumnos de actividades sorpresa, como celebraciones en clase, simulacros de incendio, etc.

4.- Explicar con anticipación los cambios que se introducirán en las rutinas.

5.- Facilitarle el acceso a espacios con los que esté familiarizado y donde se sienta seguro, tanto dentro del aula como fuera de ella.

6.- Facilitarle la interacción con personas con las que esté familiarizado y se sienta seguro.

7.- El alumno puede padecer hipo sensibilidad (baja sensibilidad) o Hipersensibilidad (sensibilidad aguda y, a veces insoportable).

8.- Percepción desigual (los niveles de sensibilidad del alumno fluctúan: pueden estar ausentes, ser muy altos o muy bajos).

9.- Dificultad para reconocer que sus ideas pueden ser diferentes de las de demás.

10.- Puede centrar su atención en los detalles sin percibir el conjunto.

11.- Puede carecer de visión de conjunto.

12.- Puede saber leer pero no comprende los textos (hiperlexia).

13.- Puede ser capaz de realizar cálculos y predicciones mentales a gran velocidad.

14.- Puede tener dificultades para elaborar un plan y seguir los pasos lógicos para alcanzar el objetivo.

15.- Puede tener dificultades para controlar sus impulsos.

16.- Puede tener dificultades para organizarse y emprender un trabajo (esto puede ser un gran obstáculo). 
17.- Puede presentar elevados niveles de ansiedad.

18.- Asignar tiempo adicional para los cambios de aula y los traslados entre las instalaciones del colegio durante períodos de gran agitación.

19.- Fomentar el hecho de que participen y se equivoquen como parte de una forma de aprender.

20.- Convertir las actividades diarias en rutinas.

21.- Proporcionar estímulos, horarios o tarjetas con textos o dibujos como apoyo al aprendizaje independiente.

22.- Utilizar los diálogos de las tiras cómicas para enseñar diversos puntos de vista.

23.- Tener expectativas ambiciosas pero realistas.

24.- Proporcionar pistas visuales para el aprendizaje.

25.- Ofrecer recompensas significativas y motivadoras.

26.- Ser predecible, coherente y fiable.

27.- Comprobar que los alumnos han comprendido lo que se les enseñó.

28.- Evitar el empleo de términos abstractos, bromas, figuras retóricas o expresiones sarcásticas.

29.- Animar a todos los integrantes del círculo social del alumno a que adopten las mismas estrategias.

30.- Brindar oportunidades para que el alumno transfiera sus habilidades a otros contextos.

31.- Crear una atmósfera de calma y orden en el aula.

32.- Proteger al niños de burlas.

33.- Si el grado del niño es severo, debemos explicar e informar a los compañeros las características de su compañero.

34.- Hacer énfasis en las habilidades académicas sobresaliente del niño.

35.- Utilizar el sistema del amigo, seleccionando un compañero que lo incluya en las actividades escolares

36.- Emplee el uso de un refuerzo positivo dirigido selectivamente para adquirir una determinada conducta correcta .

37.- Utiliza caras contentas $:-$ y caras tristes $:-$ en función de su comportamiento durante el día. Esto le da un feedback visual de lo que está haciendo. Cuenta el total de las puntuaciones y úsalo como base de un premio o un trato.

38.- Le explicamos qué ocurre si no hace lo correcto.

39. Ante roces con compañeros a anticiparse a su conducta y moldear la conducta adecuada y reforzar otras conductas incompatibles con la pelea.

40.- Aumentar la comprensión social de gestos, posturas corporales, etc. 


\section{ESCALA ASPERGER DE MEDIDA DE COMPORTAMIENTO PARA PADRES}

Ruiz (2015) publica este instrumento peruano para facilitar un primer acercamiento de toda la comunidad educativa sobre el Asperger dentro de los Trastornos del Espectro Autista, con la finalidad de que a partir de sus resultados se puedan proporcionar pautas de intervención y estrategias por parte de los profesionales y las familias que tienen contacto con este sector del alumnado dándoles la oportunidad de profundizar las buenas prácticas educativas para su inserción en la comunidad y sentirse realmente incluidos en ella.

\section{Escala Peruana para Padres en la Identificación del Síndrome de Asperger (EPPISA)}

\section{FICHA TÉCNICA}

\section{Nombre:}

La Escala Peruana para padres en la Identificación del Síndrome de Asperger por parte de los padres (EPPISA) fue elaborada por el Mg. César Ruiz Alva Psicólogo, en el 2015 dentro de los cursos de "Psicología de la Persona con Habilidades Diferentes" en la Universidad Privada del Norte y "Psicología de la Excepcionalidad" en la Universidad Antenor Orrego de Trujillo.

\section{Procedencia:}

Los ítems de la Escala han sido extraídos sobre referencias de observaciones de casuística atendida en Inclusión Educativa y también de fuentes diversas sobre el tema tales como Asperger.es ; Salud.discapnet.es y Asperger.cl/que_es_el_sindrome

\section{Propósito:}

La EPPISA ha sido diseñada para identificar por parte de los padres, 6 áreas del comportamiento que puedan ser indicativos del Síndrome de Asperger en los niños durante sus años de estudio en el colegio. Es en la edad después de los 6 años en la que se hacen más evidentes las conductas y modos inusuales de comportamiento.

\section{Áreas que evalúa:}

1.- Habilidades sociales y control Emocional.

2.. Habilidades de Comunicación.

3. Habilidades de Comprensión

4.- Intereses Específicos

5.-Habilidades de movimiento

6.- Otras características

Los niños con Síndrome de Asperger se caracterizan por tener áreas de especial interés peculiares e idiomáticas. Se interesan en áreas intelectuales específicas como las matemáticas, los temas científicos o la historia, la geografía, los mapas, la astronomía, los aviones o los trenes. Estos intereses pueden cambiar con el tiempo, pero otras veces persisten y en la edad adulta constituyen la base para una profesión. 


\section{¿CÓMO TRABAJAR LA ESCALA?}

Cada pregunta o afirmación tiene una escala de clasificación, en el que:

El 0 representa el nivel normal esperado en un escolar de primaria o secundaria y el 0 significa que el alumno no presenta ninguna anomalía cuando lo comparamos con otros chicos de su grupo de edad. Y el 5 refleja comportamientos encontrados en la persona Asperger, por lo tanto, cuánto más se aparte del 0 que es la normalidad, más nos acercaremos a la puntuación 5 que refleja la característica del cuadro Asperger.

\section{CÓMO ANALIZAR LOS DATOS?}

Cada área tiene 10 ítems con un valor máximo de puntaje por área de 50 puntos. A más puntaje más se ve reflejada la conductas Asperger aunque no se puede indicar, de manera automática, que el niño tiene el Síndrome y solamente es una tendencia a dicha posibilidad, por lo que hace necesaria la evaluación y un diagnóstico diferencial a cargo de un profesional de la salud. Solo el diagnóstico preciso y seguro orientará un eficaz tratamiento del caso.

\section{Escala Peruana para Identificar el Síndrome de Asperger por parte de los padres (EPPISA)}

(Mg. César Ruiz Alva, 2015)

Nombre y Apellido Edad Fecha de hoy

\section{AREA: I}

Habilidades sociales y control emocional

\begin{tabular}{|l|c|c|c|c|c|c|}
\hline & \multicolumn{5}{|c|}{ Raramente / A menudo } \\
\hline 1.- No disfruta normalmente del contacto social.. & 0 & 1 & 2 & 3 & 4 & 5 \\
\hline 2.- Se relaciona mejor con adultos que con los niños de su misma edad & 0 & 1 & 2 & 3 & 4 & 5 \\
\hline 3.- No se interesa por los deportes & 0 & 1 & 2 & 3 & 4 & 5 \\
\hline $\begin{array}{l}\text { 4.- Tiene problemas al jugar con otros niños. No entiende las reglas } \\
\text { implícitas del juego. }\end{array}$ & 0 & 1 & 2 & 3 & 4 & 5 \\
\hline $\begin{array}{l}\text { 5.- Quiere imponer sus propias reglas, y ganar siempre. Tal vez por eso } \\
\text { prefiera jugar sólo }\end{array}$ & 0 & 1 & 2 & 3 & 4 & 5 \\
\hline 6.- No le gusta ir al colegio. Y presenta conflictos con sus compañeros & 0 & 1 & 2 & 3 & 4 & 5 \\
\hline 7.- Le cuesta identificar sus sentimientos y de los demás. & 0 & 1 & 2 & 3 & 4 & 5 \\
\hline 8.- Presenta más rabietas de lo normal. Llora con facilidad, por todo. & 0 & 1 & 2 & 3 & 4 & 5 \\
\hline 9.- Tiene dificultades para entender las intenciones de los demás & 0 & 1 & 2 & 3 & 4 & 5 \\
\hline 10.- Es ingenuo. No tiene malicia. Es sincero & 0 & 1 & 2 & 3 & 4 & 5 \\
\hline
\end{tabular}




\section{AREA: II}

\section{Habilidades de comunicación}

\begin{tabular}{|l|c|c|c|c|c|c|}
\hline & \multicolumn{5}{|c|}{ Raramente / A menudo } \\
\hline 1.- No suele mirarte a los ojos cuando te habla. & 0 & 1 & 2 & 3 & 4 & 5 \\
\hline $\begin{array}{l}\text { 2.- Se cree en todo aquello que se le dice, y no entiende las ironías o } \\
\text { el doble sentido.. }\end{array}$ & 0 & 1 & 2 & 3 & 4 & 5 \\
\hline 3.- Se interesa poco por lo que dicen los demás & 0 & 1 & 2 & 3 & 4 & 5 \\
\hline $\begin{array}{l}\text { 4.- Le cuesta entender una conversación larga, y cambia de tema cuando } \\
\text { está confuso }\end{array}$ & 0 & 1 & 2 & 3 & 4 & 5 \\
\hline 5.- Habla mucho, en un tono alto y peculiar, & 0 & 1 & 2 & 3 & 4 & 5 \\
\hline $\begin{array}{l}\text { 6.- Inventa palabras o expresiones particulares que repite a menudo. } \\
\text { 7.- En ocasiones parece estar ausente, absorto en sus pensamientos }\end{array}$ & 0 & 1 & 2 & 3 & 4 & 5 \\
\hline $\begin{array}{l}\text { 8.- Usa un lenguaje pedante, extremamente formal y con un extenso } \\
\text { vocabulario. }\end{array}$ & 0 & 1 & 2 & 3 & 4 & 5 \\
\hline 9.- No entiende el doble sentido y piensa que se burlan de él. & 0 & 1 & 2 & 3 & 4 & 5 \\
\hline $\begin{array}{l}\text { 10.- Carece de la habilidad para el trabajo conjunto con otros como } \\
\text { el debatir un tema }\end{array}$ & 0 & 1 & 2 & 3 & 4 & 5 \\
\hline
\end{tabular}

\section{AREA: III}

\section{Habilidades de Comprensión}

\begin{tabular}{|l|c|c|c|c|c|c|}
\hline & \multicolumn{5}{|c|}{ Raramente / A menudo } \\
\hline 1.- Siente dificultad de entender el contexto amplio de un problema. & 0 & 1 & 2 & 3 & 4 & 5 \\
\hline 2.- Le cuesta entender una pregunta compleja y tarda en responder. & 0 & 1 & 2 & 3 & 4 & 5 \\
\hline 3.- A menudo no comprende una crítica o un castigo. & 0 & 1 & 2 & 3 & 4 & 5 \\
\hline $\begin{array}{l}\text { 4.. No entiende el por qué debe portarse con distintas formas, según } \\
\text { una situación social }\end{array}$ & 0 & 1 & 2 & 3 & 4 & 5 \\
\hline 5.- Tiene especial interés por las matemáticas y las ciencias en general. & 0 & 1 & 2 & 3 & 4 & 5 \\
\hline 6.- Aprende a leer solo a una edad temprana & 0 & 1 & 2 & 3 & 4 & 5 \\
\hline 7- Tiene una memoria excepcional para recordar datos y fechas. & 0 & 1 & 2 & 3 & 4 & 5 \\
\hline $\begin{array}{l}\text { 8.-- No comprende una explicación dada por el profesor en clase y } \\
\text { las anotaciones que hace suelen ser difíciles de entender. }\end{array}$ & 0 & 1 & 2 & 3 & 4 & 5 \\
\hline $\begin{array}{l}\text { 9.- Hay cursos en lo que está muy perdido aunque en otros le va } \\
\text { muy bien }\end{array}$ & 0 & 1 & 2 & 3 & 4 & 5 \\
\hline 10.- Nunca pregunta una duda ni participa. & 0 & 1 & 2 & 3 & 4 & 5 \\
\hline
\end{tabular}


AREA: IV

Intereses Específicos

\begin{tabular}{|c|c|c|c|c|c|c|}
\hline \multirow[b]{2}{*}{$\begin{array}{l}\text { 1.- Cuando algún tema en particular le fascina, ocupa la mayor parte } \\
\text { de su tiempo libre en pensar, hablar o escribir sobre el asunto, sin } \\
\text { importarse con la opinión de los demás }\end{array}$} & \multicolumn{6}{|c|}{ Raramente / A menudo } \\
\hline & 0 & 1 & 2 & 3 & 4 & 5 \\
\hline $\begin{array}{l}\text { 2.- Repite compulsivamente ciertas acciones o pensamientos para } \\
\text { sentirse seguro }\end{array}$ & 0 & 1 & 2 & 3 & 4 & 5 \\
\hline 3.- Le gusta la rutina. No tolera los cambios imprevistos. & 0 & 1 & 2 & 3 & 4 & 5 \\
\hline 4.- Tiene rituales elaborados que deben ser cumplidos & 0 & 1 & 2 & 3 & 4 & 5 \\
\hline 5.- Interesado en temas poco comunes para los chicos de su edad & 0 & 1 & 2 & 3 & 4 & 5 \\
\hline $\begin{array}{l}\text { 6.- Muestra conductas pasivas de aislarse, leer en biblioteca y lejos } \\
\text { del grupo social }\end{array}$ & 0 & 1 & 2 & 3 & 4 & 5 \\
\hline $\begin{array}{l}\text { 7.- Le es muy difícil sacar conclusiones a partir de una experiencia } \\
\text { concreta. }\end{array}$ & 0 & 1 & 2 & 3 & 4 & 5 \\
\hline $\begin{array}{l}\text { 8.- Los cambios de rutinas, esquemas y normas preestablecidas lo } \\
\text { alteran. }\end{array}$ & 0 & 1 & 2 & 3 & 4 & 5 \\
\hline $\begin{array}{l}\text { 9.- Tiene rigidez ante las normas no porque nos desafía, sino porque } \\
\text { no las entiende. }\end{array}$ & 0 & 1 & 2 & 3 & 4 & 5 \\
\hline $\begin{array}{l}\text { 10.- En la sensibilidad térmica no son muy conscientes del frío y del } \\
\text { calor por lo que no se visten de acuerdo al tiempo. }\end{array}$ & 0 & 1 & 2 & 3 & 4 & 5 \\
\hline
\end{tabular}

\section{AREA: V}

\section{Habilidades de Movimiento}

\begin{tabular}{|l|c|c|c|c|c|c|}
\hline & \multicolumn{5}{|c|}{ Raramente / A menudo } \\
\hline 1.- Posee una pobre y lenta coordinación motriz generalizada. & 0 & 1 & 2 & 3 & 4 & 5 \\
\hline $\begin{array}{l}\text { 2.- Le cuesta vestirse, abrocharse los botones o hacer un lazo con los } \\
\text { cordones de sus zapatos }\end{array}$ & 0 & 1 & 2 & 3 & 4 & 5 \\
\hline $\begin{array}{l}\text { 3.- Le resulta difícil tareas de escribir, hacer trabajos manuales o } \\
\text { aprender a tocar instrumentos musicales. }\end{array}$ & 0 & 1 & 2 & 3 & 4 & 5 \\
\hline $\begin{array}{l}\text { 4.- No gusta mucho de la actividad física y muestra poca destreza para } \\
\text { atrapar una pelota. }\end{array}$ & 0 & 1 & 2 & 3 & 4 & 5 \\
\hline 5.- Tiene reacciones motoras lentas en general. & 0 & 1 & 2 & 3 & 4 & 5 \\
\hline $\begin{array}{l}\text { 6.- Corre a un ritmo extraño y tiene una forma peculiar para andar. } \\
\text { 7.- No anota los deberes en la agenda y tampoco anota las fechas } \\
\text { de evaluaciones. }\end{array}$ & 0 & 1 & 2 & 3 & 4 & 5 \\
\hline $\begin{array}{l}\text { 8.- Tomar apuntes y sacar ideas de lo que trabaja en un tema le resulta } \\
\text { muy complicado }\end{array}$ & 0 & 1 & 2 & 3 & 4 & 5 \\
\hline $\begin{array}{l}\text { 9.- Su trazo escrito es irregular y lo ejecuta con gran esfuerzo y tensión } \\
\text { muscular. }\end{array}$ & 0 & 1 & 2 & 3 & 4 & 5 \\
\hline 10.- Le cuesta permanecer sentado escribiendo. & 0 & 1 & 2 & 3 & 4 & 5 \\
\hline
\end{tabular}


Mg. Abel Cuzcano Zapata / Mg. César Ruiz Alva-

\section{AREA: VI Otras características}

\begin{tabular}{|l|c|c|c|c|c|c|}
\hline & \multicolumn{5}{|c|}{ Raramente / A menudo } \\
\hline $\begin{array}{l}\text { 1.- Tiene miedo, angustia a sonidos como los de un aparato eléctrico } \\
\text { o ruidos intensos }\end{array}$ & 0 & 1 & 2 & 3 & 4 & 5 \\
\hline 2.-Muestra ligeros roces sobre la piel o la cabeza (manía de tocarse) & 0 & 1 & 2 & 3 & 4 & 5 \\
\hline $\begin{array}{l}\text { 3.- Hay la tendencia a agitarse o mecerse cuando está excitado o } \\
\text { angustiado }\end{array}$ & 0 & 1 & 2 & 3 & 4 & 5 \\
\hline 4.- Se le nota una falta de sensibilidad a niveles bajos de dolor & 0 & 1 & 2 & 3 & 4 & 5 \\
\hline 5.- Tiene un lenguaje demasiado pedante y correcto & 0 & 1 & 2 & 3 & 4 & 5 \\
\hline $\begin{array}{l}\text { 6.- Tiene muecas, espasmos o tics faciales inusuales } \\
\text { 7.- Tiene memoria mecánica de buen nivel y para recordar detalles. }\end{array}$ & 0 & 1 & 2 & 3 & 4 & 5 \\
\hline $\begin{array}{l}\text { 8.- Tiene rigidez cognitiva por lo que carece de iniciativa para solucionar } \\
\text { sus problemas }\end{array}$ & 0 & 1 & 2 & 3 & 4 & 5 \\
\hline $\begin{array}{l}\text { 9.- Cualquier estímulo lo puede distraer con facilidad y deja las } \\
\text { cosas a medias. }\end{array}$ & 0 & 1 & 2 & 3 & 4 & 5 \\
\hline $\begin{array}{l}\text { 10.- Cuando las actividades no le salen como quiere se molesta } \\
\text { mucho y no hay forma de controlarlo. }\end{array}$ & 0 & 1 & 2 & 3 & 4 & 5 \\
\hline
\end{tabular}




\section{REFERENCIAS}

Ainscow, M. (2005) El próximo gran reto: la mejora de la escuela inclusiva. Barcelona: España

Alonso Peña (2004) Autismo y síndrome Asperger. Salamanca, Amaru.

Attwood, A., (2002) El Síndrome de Asperger. Barcelona: Paidós.

Attwood, T., (2007) Guía del Síndrome de Asperger. Barcelona: Paidós.

Baron-Cohen, S., (2010) Autismo y Síndrome de Asperger. Madrid: Psicología Alianza Editorial.

Blanco, R. (2010) La atención educativa a la diversidad: las escuelas inclusivas. Santillana ,Madrid,

Borreguero, P., (2004) El Síndrome de Asperger. ¿Excentricidad o discapacidad social?. Madrid: Alianza Editorial.

Cohen, S., (2010) Autismo y Síndrome de Asperger. Madrid: Psicología Alianza Editorial.

Cuzcano, A (2016) El niño Asperger : estrategias de diagnóstico e intervención. Lectura del curso Psicología Educativa. Universidad Marcelino Champagnat. Lima-Perú

Kenneth Hall (2003) Soy un niño con Síndrome de Asperger. Barcelona :Paidós

Marchesi A. (2014) Avances y desafíos de la Educación inclusiva en Iberoamérica. Madrid: Fundación MAPFRE .

Ministerio de Educación (2003) Ley General de Educación. Lima Perú

Ruiz C. (2015) Escala Peruana de Asperger para padres. Perú :Universidad Privada del Norte y UPAO Trujillo.

Sans, A., (2008) ¿Por qué me cuesta tanto aprender? Trastornos del aprendizaje. Barcelona: Edebé.

Szatmari, P., (2006) Una mente diferente. Comprender a los niños con autismo y síndrome de Asperger. Barcelona: Paidós. 